\title{
HYPERBOLIC DECAY TIME SERIES
}

\author{
A.I. McLeod \\ Department of Statistical \& Actuarial Sciences \\ The University of Western Ontario \\ London, Ontario N6A 5B7 \\ Canada
}

October 1997

McLeod, A.I. (1998), Hyperbolic decay time series,

The Journal of Time Series Analysis 19, 473-484. 


\begin{abstract}
Hyperbolic decay time series such as, fractional Gaussian noise (FGN) or fractional autoregressive moving-average (FARMA) process, each exhibit two distinct types of behaviour: strong persistence or antipersistence. Beran (1994) characterized the family of strongly persistent time series. A more general family of hyperbolic decay time series is introduced and its basic properties are characterized in terms of the autocovariance and spectral density functions. The random shock and inverted form representations are derived. It is shown that every strongly persistent series is the dual of an antipersistent series and vice versa. The asymptotic generalized variance of hyperbolic decay time series with unit innovation variance is shown to be infinite which implies that the variance of the minimum mean-square error one-step linear predictor using the last $k$ observations decays slowly to the innovation variance as $k$ gets large.
\end{abstract}

Keywords. Covariance determinant; duality in time series; fractional differencing and fractional Gaussian noise; long-range dependence; minimum mean square error predictor; nonstationary time series modelling. 


\section{INTRODUCTION}

Let $Z_{t}, t=1,2, \ldots$ denote a covariance stationary, purely nondeterministic time series with mean zero and with autocovariance function, $\gamma_{Z}(k)=\operatorname{cov}\left(Z_{t}, Z_{t-k}\right)$. As is discussed by Beran (1994), many long memory processes such as the FGN (Mandelbrot, 1983) and FARMA (Granger and Joyeux, 1980; Hosking, 1981) may be characterized by the property that $k^{\alpha} \gamma_{Z}(k) \rightarrow c_{\gamma}$ as $k \rightarrow \infty$, for some $\alpha \in(0,1)$ and $c_{\gamma}>0$. Equivalently,

$$
\gamma_{Z}(k) \sim c_{\gamma} k^{-\alpha} .
$$

As noted in Box and Jenkins (1976), the usual stationary ARMA models on the other hand are exponentially damped since $\gamma_{Z}(k)=O\left(r^{k}\right), r \in(0,1)$.

Beran (1994, p.42) shows that an equivalent characterization of strongly persistent time series is

$$
f_{Z}(\lambda) \sim c_{f} \lambda^{\alpha-1} \quad \text { as } \quad \lambda \rightarrow 0,
$$

where $\alpha \in(0,1), c_{f}>0$ and $f_{Z}(\lambda)$ is the spectral density function given by $f_{Z}(\lambda)=$ $\sum \gamma_{Z}(k) e^{-i k \lambda} /(2 \pi)$. Theorem 1 below summarizes some results stated without proof in Beran (1994, Lemma 5.1). Since not all time series satisfying eq. (1) or (2) are invertible, the restriction to invertible processes is required.

THEOREM 1. The time series $Z_{t}$ satisfying (1) or (2) may be written in random shock form as $Z_{t}=A_{t}+\sum \psi_{\ell} A_{t-\ell}$, where $\psi_{\ell} \sim c_{\psi} \ell^{-(1+\alpha) / 2}, c_{\psi}>0$ and $A_{t}$ is white noise. Assuming that $Z_{t}$ is invertible, the inverted form may be written, $Z_{t}=$ $A_{t}+\sum \pi_{\ell} Z_{t-\ell}$, where $\pi_{\ell} \sim c_{\pi} \ell^{-(3-\alpha) / 2}, c_{\pi}>0$ and $A_{t}$ is white noise.

Proof. By the Wold Decomposition, any purely nondeterministic time series may be written in random shock form. Now assume the random shock coefficients specified in the theorem and we will derive (1). Assuming $\operatorname{var}\left(A_{t}\right)=1, \gamma_{Z}(k)=\psi_{k}+\sum \psi_{h} \psi_{h+k}$

$$
\begin{aligned}
\gamma_{Z}(k) & \sim \psi_{k}+c_{\psi}^{2} \sum_{h=1}^{\infty} h^{-(1+\alpha) / 2}(h+k)^{-(1+\alpha) / 2} \\
& \sim \psi_{k}+c_{\psi}^{2} \int_{1}^{\infty} h^{-(1+\alpha) / 2}(h+k)^{-(1+\alpha) / 2} d h+R_{k}
\end{aligned}
$$


where the last step used the Euler summation formula (Graham, Knuth and Patashnik, $1989,9.78,9.80)$ and

$$
R_{k}=\left.\left\{-\frac{1}{2} F(h)+\frac{1}{12} F^{\prime}(h)+\frac{\theta}{720} F^{\prime \prime \prime}(h)\right\}\right|_{1} ^{\infty}
$$

where $\theta \in(0,1)$ and $F(h)=h^{-(1+\alpha) / 2}(h+k)^{-(1+\alpha) / 2}$. It is easily shown that $k^{\alpha} R_{k} \rightarrow 0$ as $k \rightarrow \infty$. Hence,

$$
\begin{aligned}
\gamma_{Z}(k) & \sim \psi_{k}+c_{\psi}^{2} \int_{1}^{\infty} h^{-(1+\alpha) / 2}(h+k)^{-(1+\alpha) / 2} d h \\
& \sim \psi_{k}+k^{-\alpha} c_{\psi}^{2} \int_{1 / k}^{\infty} x^{-\beta}(x+1)^{-\beta} d x
\end{aligned}
$$

where $\beta=(1+\alpha) / 2$. Using Mathematica,

$$
\int_{0}^{\infty} x^{-\beta}(x+1)^{-\beta} d x=\frac{2^{2 \beta} \Gamma(1-\beta) \Gamma\left(-\frac{1}{2}+\beta\right)}{4 \sqrt{\pi}},
$$

so (1) now follows with $c_{\gamma}=c_{\psi}^{2} 2^{\alpha-1} \Gamma((1-\alpha) / 2) \Gamma(\alpha / 2) / \sqrt{\pi}$, where $\Gamma(\bullet)$ is the gamma function. This shows that $\psi_{k}$ is a possible factorization of $\gamma_{k}$ and that sufficies to establish that $Z_{t}=A_{t}+\sum \psi_{\ell} A_{t-\ell}$.

For any stationary invertible linear process, $Z_{t}$,

$$
\gamma_{Z}(k)=\sum_{h=1}^{\infty} \pi_{h} \gamma_{Z}(k-h)
$$

Assume $\gamma_{Z}(k)$ satisfies eq. (1) and that $\pi_{\ell} \sim c_{\pi} \ell^{-(3-\alpha) / 2}$ then we will show that eq. (3) is satisfied.

$$
\gamma_{Z}(k) \sim \gamma_{Z}(0) \pi_{k}+c \sum_{h=1}^{k-1} h^{-3 / 2+\alpha / 2}(k-h)^{-\alpha}+c \sum_{h=k+1}^{\infty} h^{-3 / 2+\alpha / 2}(h+k)^{-\alpha},
$$

where $c=c_{\pi} c_{\gamma}$. Now $\gamma_{Z}(0) \pi_{k} / \gamma_{Z}(k) \sim 0$ so the first term will drop out. In the second term, for $k>>h,(k-h)^{-\alpha} \sim k^{-\alpha}$ and

$$
\sum_{h=1}^{k-1} h^{-3 / 2+\alpha / 2} \sim H_{\beta} \quad \text { as } \quad k \rightarrow \infty
$$


where $H_{\beta}=\sum_{h=1}^{\infty} h^{-\beta}<\infty, \beta=3 / 2-\alpha / 2$. In the final term, when $h>>k,(h+k)^{-\alpha} \sim$ $h^{-\alpha}$, so

$$
\begin{aligned}
\sum_{h=k+1}^{\infty} h^{-3 / 2+\alpha / 2}(h+k)^{-\alpha} & \sim \sum_{h=k+1}^{\infty} h^{-3 / 2-\alpha / 2} \\
& \sim \int_{k+1}^{\infty} h^{-3 / 2-\alpha / 2} d h \\
& \sim(k+1)^{-(1+\alpha) / 2} .
\end{aligned}
$$

Again the last step uses the Euler Summation Formula. Thus the final term is smaller asymptotically smaller than $\gamma_{k}$. This establishes the asymptotic equivalence of the lefthand side and the right-hand side of eq. (3) and the theorem since $\gamma_{Z}(k)$ uniquely determines the coefficients $\pi_{\ell}$ in the inverted model. $\diamond$

The FARMA model of order $(p, q)$ (Granger and Joyeux, 1980; Hosking, 1981) may be defined by the equation,

$$
\phi(B)(1-B)^{d} Z_{t}=\theta(B) A_{t}
$$

where $|d|<0.5, A_{t}$ is white noise with variance $\sigma_{A}^{2}, \phi(B)=1-\phi_{1} B-\ldots-\phi_{p} B^{p}$, and $\theta(B)=1-\theta_{1} B-\ldots-\theta_{q} B^{q}$. For stationarity and invertibility it is assumed that all roots of $\phi(B) \theta(B)=0$ are outside the unit circle and $|d|<0.5$. The series is strongly persistent or antipersistent according as $0<d<0.5$ or $-0.5<d<0$. The special case where $p=q=0$ is known as fractionally differenced white noise.

Antipersistent series may arise in practice when modelling nonstationary time series. As suggested by Box \& Jenkins (1976) a nonstationary time series can often be made stationary by differencing the series until stationarity is reached. Sometimes the resulting stationary time series may be usefully modelled by an antipersistent form of the FARMA model. An illustrative example is provided by the annual U.S. electricity consumption data for 1920-1970. Hipel and McLeod (1994, pp.154-159) modelled the square-root consumption using an $\operatorname{ARIMA}(0,2,1)$ but a better fit is obtained by modelling the second differences of the square-root consumption as fractionally differenced 
white noise with $d=-0.4477 \pm 0.1522 \mathrm{sd}$. The AIC for the latter model is 1011.5 as compared with 1020.4. Diebold and Rudebusch (1989) and Beran (1995) also used this approach for modelling nonstationary data.

The determinant of the covariance matrix of $n$ successive observations, $Z_{t}, t=$ $1, \ldots, n$, is denoted by $G_{Z}(n)=\operatorname{det}\left(\gamma_{Z}(i-j)\right)$. It will now be shown in Theorem 2 that for fractionally differenced white noise, $g_{Z}(n)=\sigma_{A}^{-2 n} G_{Z}(n) \rightarrow \infty$ as $n \rightarrow \infty$, where $0<\sigma_{A}^{2}<\infty$, is the innovation variance given by Kolmogoroff's formula (Brockwell and Davis, eq. 5.8.1). In Theorems 7, 8 and 9 this result will be established for a more general family of processes. Since $g_{Z}(n)$ is the generalized variance of the process $Z_{t} / \sigma_{A}$, it will be referred to as the standardized generalized variance. Without loss of generality we will let $\sigma_{A}=1$.

THEOREM 2. Let $Z_{t}$ denote fractionally differenced white noise with parameter $d \in$ $\left(-\frac{1}{2}, \frac{1}{2}\right)$ and $d \neq 0$. Then $g_{Z}(n) \rightarrow \infty$.

Proof. As in McLeod (1978), $g_{Z}(n)=\prod_{k=0}^{n-1} \sigma_{k}^{2}$, where $\sigma_{k}^{2}$ denotes the variance of the error in the linear predictor of $Z_{k+1}$ using $Z_{k}, \ldots, Z_{1}$. From the Durbin-Levinson recursion,

$$
\sigma_{k}^{2}= \begin{cases}\gamma_{Z}(0) & k=0 \\ \sigma_{k-1}^{2}\left(1-\phi_{k, k}^{2}\right) & k>0\end{cases}
$$

where $\phi_{k, k}$ denotes the partial autocorrelation function at lag $k$. For the special case $p=$ $q=0$ in (4), Hosking (1981) showed that $\phi_{k, k}=d /(k-d)$ and $\gamma_{Z}(0)=(-2 d) ! /(-d) !^{2}$. Using the Durbin-Levison recursion,

$$
\sigma_{k}^{2}=\frac{k !(k-2 d) !}{(k-d) !^{2}}
$$

Applying the Stirling approximation to $\log (t !)$ for large $t, \log (t !) \sim\left(t+\frac{1}{2}\right) \log (t)-t+$ $\frac{1}{2} \log (2 \pi)$, yields $\log \sigma_{k}^{2} \sim a(k)$, where

$$
a(k)=\left(k+\frac{1}{2}\right) \log \frac{k(k-2 d)}{(k-d)^{2}}+2 d \log \frac{k-d}{k-2 d} .
$$


Since $\sigma_{k}^{2}$, is a monotone decreasing sequence and for $d \neq 0, \sigma_{k}^{2}>1$, it follows that $\log \left(\sigma_{k}^{2}\right)$ is a positive monotone decreasing sequence. By Stirling's approximation $\log \left(\sigma_{k}^{2}\right) / a(k) \rightarrow 1$ as $k \rightarrow \infty$. So for large $k, a(k)$ must be a monotone decreasing sequence of positive terms. Expanding $a(k)$ and simplifying

$$
\begin{aligned}
a(k) & =\left(k+\frac{1}{2}\right) \log \left(1-\frac{2 d}{k}\right)+2\left(k+\frac{1}{2}\right) \log \left\{1+\frac{d}{k}+\left(\frac{d}{k}\right)^{2}+\ldots\right\}+2 d \log \left(1+\frac{d}{k-2 d}\right) \\
& =\frac{d^{2}}{k}+O\left(\frac{1}{k^{2}}\right),
\end{aligned}
$$

where the expansion $\log (1+x)=x+x^{2} / 2+x^{3} / 3+\ldots,|x|<1$ as been used. Hence,

$$
k a(k) \rightarrow d^{2} \text { as } k \rightarrow \infty
$$

and by the Theorem given by Knopp (1951, §80, p.124), $\sum a(k)$ diverges for $d \neq 0$. So for $d \neq 0, \sum \log \left(\sigma_{k}^{2}\right)$ diverges and consequently so does $g_{Z}(n)$. $\diamond$

Eq. (5) shows that $\sigma_{k}^{2}=1+O\left(k^{-1}\right)$ which implies $\sigma_{k}^{2}$ decays very slowly. The divergence of $g_{Z}(n)$ can be slow. See Table I.

TABLE I.

Generalized VARIANCE, $g_{Z}(n)$, FOR $n=10^{k}, k=0,1, \ldots, 7$

OF FRACTIONALLY DifFERENCED White NOISE, $Z_{t}$, With PARAMETER $d$.

\begin{tabular}{cccrrrrrr}
\hline \hline$d$ & $k=0$ & $k=1$ & $k=2$ & $k=3$ & $k=4$ & $k=5$ & $k=6$ & $k=7$ \\
\hline-0.4 & 1.1831 & 1.6225 & 2.3318 & 3.3685 & 4.8688 & 7.0375 & 10.1725 & 14.7059 \\
-0.1 & 1.0145 & 1.0366 & 1.0607 & 1.0854 & 1.1107 & 1.1365 & 1.1630 & 1.1901 \\
0.1 & 1.0195 & 1.0434 & 1.0678 & 1.0927 & 1.1181 & 1.1442 & 1.1708 & 1.1990 \\
0.4 & 2.0701 & 3.1588 & 4.5923 & 6.6417 & 9.6009 & 13.8775 & 20.0591 & 28.9951
\end{tabular}

\section{HYPERBOLIC DECAY TIME SERIES}


The stationary, purely nondeterministic time series, $Z_{t}$, is said to be a hyperbolic decay time series with decay parameter $\alpha, \alpha \in(0,2), \alpha \neq 1$, if for large $k$

$$
\gamma_{Z}(k) \sim c_{\gamma} k^{-\alpha}
$$

where $c_{\gamma}>0$ for $\alpha \in(0,1)$ and $c_{\gamma}<0$ for $\alpha \in(1,2)$. When $\alpha \in(1,2)$ the time series is said to be antipersistent. As shown in the next theorem, antipersistent time series have a spectral density function which decays rapidly to zero near the origin. The term antipersistent was coined by Mandelbrot (1983) for FGN processes with Hurst parameter, $0<H<1 / 2$. Hyperbolic decay time series include both FGN time series with parameter $H=1-\alpha / 2, H \in(0,1), H \neq 1 / 2$ and FARMA time series with parameter $d=1 / 2-\alpha / 2, d \in(-1 / 2,1 / 2), d \neq 0$.

THEOREM 3. The spectral density function of hyperbolic decay time series satisfies (2).

Proof. Beran (1994) established this result when $\alpha \in(0,1)$ as was noted above in eq. (2). However the Theorem of Zygmund (1968, §V.2) used by Beran (1994, Theorem 2.1) does not apply to the case where $\alpha \in(1,2)$.

Let $Y_{t}$ have the spectral density, $f_{Y}(\lambda)=c_{f} \lambda^{\alpha-1}, \quad \alpha \in(1,2)$.

$$
\begin{aligned}
\gamma_{Y}(k) & =2 \int_{0}^{\pi} c_{f} \lambda^{\alpha-1} \cos (\lambda k) d \lambda \\
& =2 c_{f} k^{-\alpha} \int_{0}^{k \pi} u^{\alpha-1} \cos (u) d u
\end{aligned}
$$

Using Mathematica,

$$
\int_{0}^{\infty} u^{\alpha-1} \cos (u) d u=\frac{\sqrt{\pi} \Gamma\left(\frac{\alpha}{2}\right)}{\left(\frac{1}{4}\right)^{\frac{\alpha-1}{2}} \Gamma\left(\frac{1-\alpha}{2}\right)}
$$

and so $\gamma_{Y}(k) \sim c_{\gamma} k^{-\alpha}$, where $c_{\gamma}=2 c_{f} \sqrt{\pi} \Gamma\left(\frac{\alpha}{2}\right) /\left\{\left(\frac{1}{4}\right)^{\frac{\alpha-1}{2}} \Gamma\left(\frac{1-\alpha}{2}\right)\right\}<0$.

Assume $f_{Z}(\lambda)$ satisfies eq. (2) and we will derive (6). Since $f_{Z}(\lambda) /\left(c_{f} \lambda^{\alpha-1}\right) \rightarrow 1$ as $\lambda \rightarrow 0$, there exists $\lambda_{0}$ such that for all $\lambda<\lambda_{0}, c_{f} \lambda^{\alpha-1}<1$ and $\left|f_{Z}(\lambda) /\left(c_{f} \lambda^{\alpha-1}\right)-1\right|<$ 
$\epsilon /(2 \pi)$. Hence for all $\lambda<\lambda_{0},\left|f_{Z}(\lambda)-f_{Y}(\lambda)\right|<\epsilon /(2 \pi)$. Consider the systematically sampled series, $Z_{t, \ell}=Z_{t \ell}$ for $\ell \geq 1$. Then $Z_{t, \ell}$ has spectral density function, $f_{Z}(\lambda / \ell)$. Let $L=\pi / \lambda_{0}$. Then $\left|f_{Z}(\lambda / \ell)-f_{Y}(\lambda)\right|<\epsilon /(2 \pi)$ for $\lambda \in(0, \pi)$ provided that $\ell>L$. Hence for any $\ell>L$,

$$
\begin{aligned}
\left|\gamma_{Z}(k l)-\gamma_{Y}(k)\right| & <2 \int_{0}^{\pi}|\cos (\lambda k)|\left|f_{Z}(\lambda / \ell)-f_{Y}(\lambda)\right| d \lambda \\
& <2 \int_{0}^{\pi}\left|f_{Z}(\lambda / \ell)-f_{Y}(\lambda)\right| d \lambda \\
& <\epsilon .
\end{aligned}
$$

This shows (2) implies (6). Since the spectral density uniquely defines the autocovariance function, the theorem follows. $\diamond$

Hyperbolic decay time series are self-similar: aggregated series are hyperbolic with the same parameters as the original.

Theorem 4. Let $Z_{t}$ satisfy eq. (6) then so does $Y_{t}$, where $Y_{t}=\sum_{j=1}^{m} Z_{(t-1) m+j} / m$ and $m$ is any value.

ProOF. For large $\ell$,

$$
\begin{aligned}
\gamma_{Y}(\ell) & =m^{-2} \operatorname{cov}\left(\sum_{h=1}^{m} Z_{(t-1) m+h}, \sum_{k=1}^{m} Z_{(t-1+\ell) m+k}\right) \\
& \sim m^{-2} \sum_{h=1}^{m} \sum_{k=1}^{m} c_{\gamma}(k+m \ell-h)^{-\alpha} \\
& \sim m^{-2} \sum_{h=1}^{m} \sum_{k=1}^{m} c_{\gamma}^{\prime} \ell^{-\alpha}\left(1+\frac{(k-h)}{m \ell}\right)^{-\alpha} \\
& \sim c_{\gamma}^{\prime} \ell^{-\alpha}
\end{aligned}
$$

where $c_{\gamma}^{\prime}=m^{-\alpha} c_{\gamma} \cdot \diamond$

\section{DUALITY}

Duality has provided insights into linear time series models (Finch, 1960; Pierce, 1970; Cleveland, 1972; Box and Jenkins, 1976; Shaman, 1976; McLeod, 1977, 1984). In 
general, the dual of the stationary invertible linear process $Z_{t}=\psi(B) A_{t}$ is defined to be $\psi(B) \ddot{Z}_{t}=A_{t}$, where $\psi(B)=1+\psi_{1} B+\psi_{2} B^{2}+\ldots$ and $B$ is the backshift operator on $t$. Equivalently, if $Z_{t}$ has spectral density $f_{Z}(\lambda)$ then the dual has spectral density proportional to $1 / f_{Z}(\lambda)$ with the constant of proportionality determined by the innovation variance. Thus in the case of $\operatorname{FARMA}(p, q)$ with parameter $d$ the dual is a $\operatorname{FARMA}(q, p)$ with parameter $-d$. The next theorem generalizes this to the hyperbolic case.

THEOREM 5. The dual of a hyperbolic decay time series with decay parameter $\alpha$ is another hyperbolic decay series with parameter decay parameter $2-\alpha$.

Proof. The spectral density near zero of the dual of a hyperbolic decay time series with parameter $\alpha$ is $1 /\left(c_{f} \lambda^{\alpha-1}\right)=c_{f}^{-1} \lambda^{(2-\alpha)-1}$ which implies a hyperbolic process with parameter $2-\alpha . \diamond$

THEOREM 6. The time series $Z_{t}$ satisfying (6) may be written in random shock form as $Z_{t}=A_{t}+\sum \psi_{\ell} A_{t-\ell}$ where $\psi_{\ell} \sim c_{\psi} \ell^{-(1+\alpha) / 2}$ and $c_{\psi}>0$ for $\alpha \in(0,1)$ and $c_{\psi}<0$ for $\alpha \in(1,2)$ and in inverted form as $Z_{t}=A_{t}+\sum \pi_{\ell} Z_{t-\ell}$ where $\pi_{\ell} \sim c_{\pi} \ell^{-(3-\alpha) / 2}$ and $c_{\pi}>0$ for $\alpha \in(0,1)$ and $c_{\pi}<0$ for $\alpha \in(1,2)$

Proof. The case $\alpha \in(0,1)$ was established in Theorem 1 . When $\alpha \in(1,2)$ the random shock coefficients are given by

$$
\begin{aligned}
\psi_{\ell} & \sim-c_{2-\pi} \ell^{-\{3-(2-\alpha)\} / 2} \\
& \sim c_{\psi} \ell^{-(1+\alpha) / 2}
\end{aligned}
$$

where $c_{\psi}=-c_{2-\pi}$. Similarly for the inverted form. $\diamond$

\section{GENERALIZED VARIANCE}

For ARMA processes, $Z_{t}, \lim g_{Z}(n)$ is finite and has been evaluated by Finch (1960) and McLeod (1977). McLeod (1977, eq. 2) showed $g_{Z}(n)=m_{Z}+O\left(r^{n}\right)$, where $r \in(0,1)$. The evaluation of this limit uses the Theorem of Grenander and Szegö $(1984, \S 5.5)$ 
which only applies to the case where the spectral density, $f_{Z}(\lambda), \lambda \in[0,2 \pi)$ satisfies the Lipschitz condition $\left|f_{Z}^{\prime}\left(\lambda_{1}\right)-f_{Z}^{\prime}\left(\lambda_{2}\right)\right|<K\left|\lambda_{1}-\lambda_{2}\right|^{\zeta}$, for some $K>0$ and $0<\zeta<1$. Since when $\alpha \in(0,1), f_{Z}^{\prime}(\lambda)$ is unbounded, this condition is not satisfied.

LEMma 1. Let $X_{t}$ and $Y_{t}$ be any independent stationary processes with positive innovation variance and let $Z_{t}=X_{t}+Y_{t}$. Then $G_{Z}(n)>G_{X}(n)$

ProOF. This follows directly from the fact that the one-step predictor error variance of $Z_{t}$ can not be less than that of $X_{t}$ •

THEOREM 7. Let $Z_{t}$ denote a strongly persistent time process defined in eq. (2). Then $g_{Z}(n) \rightarrow \infty$.

Proof. Since $Z_{t}=\sum \psi_{k} A_{t-k}$, where $A_{t}$ is white noise with unit variance, we can find a $q$ such that the process $Y_{t}$, where

$$
Y_{t}=\sum_{k=q+1}^{\infty} \psi_{k} A_{t-k}
$$

has all autocovariances nonnegative and satisfying eq. (1). By using the comparison test for a harmonic series, it must be possible to find an $N$ such that for $n>N$, the covariance matrix $\Gamma_{Y}(n)$ has every row-sum greater than $\Xi$, for any $\Xi>0$. It then follows from Frobenius Theorem (Minc and Marcus, 1964, p.152) that the largest eigenvalue of $\Gamma_{Y}(n)$ tends to $\infty$ as $n \rightarrow \infty$. Assume now that inf $f_{Y}(\lambda)=m$ where $m>0$ and let $m_{n}$ denote the smallest eigenvalue of $\Gamma_{Y}(n)$ and let $\zeta_{n}$ denote the corresponding eigenvector. Then

$$
\begin{aligned}
m_{n} & =m_{n} \zeta_{n}^{\prime} \zeta_{n} \\
& =\zeta_{n}^{\prime} \Gamma_{Y}(n) \zeta_{n} \\
& =\int_{-\pi}^{\pi} \sum_{h} \sum_{\ell} \zeta_{n, h} \zeta_{n, \ell} e^{-i \lambda(h-\ell)} f(\lambda) d \lambda \\
& \geq 2 \pi m .
\end{aligned}
$$

So $m_{n} \geq 2 \pi m$ and hence $g_{Y}(n) \rightarrow \infty$ as $n \rightarrow \infty$. By Lemma $1, g_{Z}(n) \rightarrow \infty$ also. 
For the more general case where $m=0$, consider a process with spectral density function $f(\lambda)+\epsilon$, where $\epsilon>0$. Let $g_{\epsilon}(n)$ denote the standardized covariance determinant of $n$ successive observations of this process. So $g_{\epsilon}(n) \rightarrow \infty$ as $n \rightarrow \infty$ for every $\epsilon>0$. The autocovariance function corresponding to $f(\lambda)+\epsilon$ is

$$
\gamma_{\epsilon}(k)= \begin{cases}\gamma_{Z}(0)+2 \pi \epsilon & k=0 \\ \gamma_{Z}(k) & k \neq 0\end{cases}
$$

By continuity of the autocovariance function with respect to $\epsilon, \lim g_{\epsilon}(n) \rightarrow g_{Z}(n)$ as $\epsilon \rightarrow 0$. Let $\Xi>0$ be chosen as large as we please and let $\delta>0$. Then for any $\epsilon>0$ there exists an $N(\epsilon)$ such that for all $n \geq N(\epsilon), g_{\epsilon}(n)>\Xi+\delta$. By continuity, there exists an $\epsilon_{0}$ such that $g_{Z}\left(N\left(\epsilon_{0}\right)\right)>g_{\epsilon_{0}}\left(N\left(\epsilon_{0}\right)\right)-\delta$. Hence $g_{Z}\left(N\left(\epsilon_{0}\right)\right)>\Xi$. Since $g_{Z}(n+$ $1)=g_{Z}(n) \sigma_{n}^{2}$, where $\sigma_{n}^{2}>1$ is the variance of the error of the linear predictor of $Z_{n+1}$ given $Z_{n}, \ldots, Z_{1}$ we see that $g_{Z}(n)$ is nondecreasing. It follows that $g_{Z}(n)>\Xi$ for all $n>N\left(\epsilon_{0}\right) . \diamond$

Using a Theorem of Grenander and Szegö (1984) this result is easily generalized to any stationary time series, $Z_{t}$, for which $\sum \gamma_{Z}(k)=\infty$.

TheOREM 8. Let $Z_{t}$ denote a time series for which $f_{Z}(\lambda) \rightarrow \infty$ as $\lambda \rightarrow 0$. Then $g_{Z}(n) \rightarrow \infty$

Proof. From eq. (10) of Grenander and Szegö (1984, §5.2), as $n \rightarrow \infty$, the largest eigenvalue of $\sigma_{a}^{-2} \Gamma_{Z}(n)$ approaches $\sup f_{Z}(\lambda)=\infty$ while the smallest eigenvalue approaches $2 \pi m$, where $m=\inf f(\lambda)$. Note that Grenander and Szegö's eq. (10) of $§ 5.2$, applies directly to unbounded spectral densities as is pointed by Grenander and Szegö in the sentence immediately following eq. (10), §5.2. If it is assumed that $m>0$, then the largest eigenvalue tends to infinity and the smallest one is bounded by $2 \pi m$ as $n \rightarrow \infty$. Hence, $g_{Z}(n) \rightarrow \infty$ for this special case. The more general case where $m=0$ is handled as in Theorem $7 . \diamond$ 
In the case of ARMA models, the asymptotic covariance determinant of the dual and primal are equal (Finch, 1960). Since the hyperbolic decay time series are approximated by high order AR and MA models, it might be expected that this property holds for hyperbolic series too. Theorem 9 which uses Lemma 2 proves that this is the case.

Lemma 2. Let $X_{t}=A_{t}+\sum_{1}^{\infty} \psi_{\ell} A_{t-\ell}$. Let $X_{t}(q)=A_{t}+\sum_{1}^{q} \psi_{\ell} A_{t-\ell}$, and let $g_{q}(n)$ denote its standardized covariance determinant. Then for any $\ell>0, g_{q+\ell}(n) \geq g_{q}(n)$.

PROOF. This follows directly from the fact that the one-step predictor error variance of $X_{t}(q+\ell)$ can not be less than that of $X_{t}(q) . \diamond$

TheOREM 9. For hyperbolic decay antipersistent time series, $Z_{t}, g_{Z}(n) \rightarrow \infty$.

ProOF. Since the dual of the antipersistent time series $Z_{t}$ with parameter $2-\alpha, \alpha \in(0,1)$ is a strongly persistent time series $\ddot{Z}_{t}$ with parameter $\alpha, \ddot{Z}_{t}$ may be represented in inverted form, $\ddot{Z}_{t}=A_{t}+\sum \pi_{k} \ddot{Z}_{t-k}$, where $A_{t}$ is white noise and for large $k, \pi_{k} \sim c_{\pi} k^{-(3-\alpha) / 2}$. So the antipersistent time series $Z_{t}$ can be written, $Z_{t}=A_{t}-\sum \pi_{k} A_{t-k}$. Let $\ddot{g}_{L}(n)$ and $g_{L}(n)$ denote the covariance determinant of $n$ successive observations in the $\operatorname{AR}(L)$ and $\operatorname{MA}(L)$ approximation to $\ddot{Z}_{t}$ and $Z_{t}$

$$
\ddot{Z}_{t}(L)=A_{t}+\sum_{k=1}^{L} \pi_{k} \ddot{Z}_{t-k}(L)
$$

and

$$
Z_{t}(L)=A_{t}-\sum_{k=1}^{L} \pi_{k} A_{t-k} .
$$

By Theorem 7, for any $\Xi>0$ and $\delta>0$ there exists an $N_{1}$ such that for $n>N_{1}$, $g_{\ddot{Z}}(n)>\Xi+\delta$. Since $\ddot{g}_{k}(n) \rightarrow \ddot{g}_{Z}(n)$ as $k \rightarrow \infty$ there exists a $K_{1}(n)$ such that $\ddot{g}_{k}(n)>$ $\ddot{g}_{Z}(n)-\delta>\Xi$ for $k>K_{1}(n)$. From McLeod (1977), $\ddot{g}_{k}(n)=\ddot{g}_{k}(k)$ for $n \geq k$. Hence for any $n>N_{1}, \ddot{g}_{k}(m)>\ddot{g}_{Z}(n)-\delta>\Xi$ for $k>K_{1}(n)$ and $m \geq k$. So $\ddot{g}_{k}(m) \rightarrow \infty$ as $k \rightarrow \infty$ and $m \geq k$.

Hence there exists $K_{2}$ such that $\ddot{g}_{k}(n)>\Xi+\delta$ for $k>K_{2}$ and $n \geq k$. For any $k$, $g_{k}(n)=\ddot{g}_{k}(n)+O\left(r^{n}\right)$, where $0<r<1\left(\right.$ McLeod, 1977). Let $k>K_{2}$. Then there exists 
an $N_{2}(k)$ such that for all $n>N_{2}(k), g_{k}(n)>\ddot{g}_{k}(n)-\delta>\Xi$. So $g_{k}(n) \rightarrow \infty$ as $k \rightarrow \infty$ and $n \geq k$.

For any $n, g_{k}(n) \rightarrow g_{Z}(n)$ as $k \rightarrow \infty$. So for any $n$ there exists a $K_{3}(n)$ such that $g_{Z}(n)>g_{k}(n)-\delta$ for all $k>K_{3}(n)$. We have already established that there exists a $K_{4}$ such that $g_{k}(n)>\Xi+\delta$ for $k>K_{4}$ and $n \geq k$. Holding $n$ fixed for the moment, let $h>k$. By Lemma $2, g_{h}(n) \geq g_{k}(n)$. By continuity since $h>K_{4}, g_{Z}(n)>g_{h}(n)-\delta$. Since $g_{h}(n)>\Xi+\delta$ it follows that $g_{Z}(n)>\Xi$. This establishes that $g_{Z}(n) \rightarrow \infty$ as $n \rightarrow \infty \cdot \diamond$

\section{CONCLUDING REMARKS}

Theorems 7 and 9 show that hyperbolic decay time series, even antipersistent ones, exhibit a type of long-range dependence. The asymptotic standardized generalized variance is infinite. This implies that the variance of the one-step linear predictor based on the last $k$ observations decays very slowly as compared with the ARMA case where the decay to the innovation variance occurs exponentially fast. Theorem 8 shows that this is a more general notion of long-range dependence than the customary one.

Yakowitz and Heyde (1997) show that nonlinear Markov processes can also exhibit strongly persistent hyperbolic decay in the autocorrelation function. Hence a better term for long-memory time series might be strongly persistent hyperbolic decay series. It is then clear that the long-range dependent aspect is merely a characterization of the autocorrelation structure.

\section{REFERENCES}

Beran, J. (1994). Statistics for Long Memory Processes. London: Chapman and Hall. Beran, J. (1995). Maximum likelihood estimation of the differencing parameter for invertible short and long memory autoregressive integrated moving average models. Journal of the Royal Statistical Society B 57, 659-672. 
Box, G.E.P. And Jenkins, G.M. (1976). Time Series Analysis: Forecasting and Control. (2nd edn). San Francisco: Holden-Day.

Brockwell, P.J. And Davis, R.A. (1991). Time Series: Theory and Methods. (2nd edn.). New York: Springer-Verlag.

Cleveland, W.S. (1972). The inverse autocorrelations of a time series and their applications. Technommetrics 14, 277-293.

Diebold, F.X. \& Rudebusch, G.D. (1989). Long memory and persistence in aggregate output. Journal of Monetary Economics, 24, 189-209.

Finch, P.D. (1960). On the covariance determinants of autoregressive and moving average models. Biometrika 47, 194-196.

Graham, R.L., Knuth, D.E. and Patashnik, O. (1989). Concrete Mathematics. Reading: Addison-Wesley.

Granger, C.W.J. And Joyeux, R. (1980). An introduction to long-range time series models and fractional differencing. Journal of Time Series Analysis 1, 15-30.

Grenander, U. And Szegö, G. (1984). Toeplitz Forms and Their Applications, (2nd edn.). New York: Chelsea.

Hipel, K.W. \& MCLeOD, A.I. (1994). Time Series Modelling of Water Resources and Environmental Systems. Elsevier: Amsterdam.

Hosking, J.R.M. (1981). Fractional differencing. Biometrika 68, 165-176.

Knopp, K. (1951). Theory and Application of Infinite Series, (2nd edn.). New York: Hafner.

Mandelbrot, B.B.M. (1983). The Fractal Geometry of Nature. San Francisco: Freeman.

MCLeOD, A.I. (1977). Improved Box-Jenkins estimators. Biometrika 64, 531-534.

MCLeOD, A.I. (1984). Duality and other properties of multiplicative seasonal autoregressive-moving average models. Biometrika 71, 207-211. 
Minc, H. And Marcus, M (1964). A Survey of Matrix Theory and Matrix Inequalities. Boston: Prindle, Weber and Schmidt.

Pierce, D.A. (1970). A duality between autoregressive and moving average processes concerning their parameter estimates. Annals of Statistics 41, 722-726.

Shaman, P. (1976). Approximations for stationary covariance matrices and their inverses with application to ARMA models. Annals of Statistics 4, 292-301.

Yakowitz, S.J. And Heyde, C.C. (1997). Long-range dependency effects with implications for forecasting and queueing inference. Unpublished Manuscript.

Zygmund, A. (1968). Trigonometric Series. London: Cambridge University Press. 